\title{
Role of immunoglobulin G antibodies in diagnosis of food allergy
}

\author{
Jacek Gocki, Zbigniew Bartuzi
}

Department of Allergology, Clinical Immunology and Internal Diseases, Ludwik Rydygier Collegium Medicum in Bydgoszcz, Nicolaus Copernicus University in Torun, Poland

Adv Dermatol Allergol 2016; XXXIII (4): 253-256

DOI: 10.5114/ada.2016.61600

\begin{abstract}
This paper presents current views on the role of immunoglobulin $\mathrm{G}(\operatorname{lgG})$ antibodies in the reactions with food antigens in the digestive tract and their role in the diagnosis of food allergy based on the assays of specific lgG class antibodies, with a special focus on contemporary practice guidelines. In the light of current scientific knowledge, the IgG-specific antibody-mediated reactions are a body's natural and normal defensive reactions to infiltrating food antigens, which are considered as pathogens. On the other hand, specific lgG antibodies against food allergens play a crucial role in the induction and maintaining of immunological tolerance to food antigens. The statements of many scientific societies stress that slgG are of no significant importance in the diagnosis of food allergy since their presence is associated with a normal immune response to food allergens and attests to a protracted exposure to food antigens.
\end{abstract}

Key words: immunoglobulin G, food allergy, diagnosis.

The role of immunoglobulin $\mathrm{G}(\mathrm{lgG})$ antibodies in the diagnosis of food allergy was already discussed and studied many years ago. It was determined then that they do not play a causal role in eliciting food hypersensitivity reactions [1]. Type III hypersensitivity reactions, associated with IgG antibody-mediated response, are a body's normal reactions to food antigens, which are usually absorbed into the bloodstream in small quantities. Healthy people produce and maintain high IgG antibody titres against food antigens. After a meal, there are both antibodies and complexes of food antigens bound to specific lgGs circulating in the serum. These complexes are quickly cleared by the reticuloendothelial system, which is why their pathogenic significance is negligible. Whereas the presence of excess antigen or antibodies may lead to the immune complexes being deposited in the blood vessels of the skin, kidneys, and joints (serum disease and Arthus-type reactions). Clinical observations suggested that IgGs involved in type III reactions may initiate some adverse food reactions and, by contributing to increased cuticular permeability, they may also play a role in the pathogenesis of chronic intestinal inflammations [1].

It is these observations along with the fact that in some food hypersensitivity cases it is not possible to demonstrate the causal role of classic immune and non-immune reactions that lead to some scholars being interested in lgG-mediated reactions. This interest is so strong that commercial tests to titrate allergen-specific IgG antibodies directed against food antigens were prepared. The results of these tests are difficult to be interpreted for both medical practitioners and patients themselves as they show the presence of specific IgG antibodies against foods often eaten by the patient. Dietary recommendations based on these test results advise that these foods should be avoided, which is even more confusing to the patients. The difficulties in the interpretation of these tests, which often fail to determine IgG subclasses directed against food antigens, prompted the authors to analyse the role of IgG antibodies in causing food hypersensitivity in the light of modern scientific research.

\section{Contact between food allergens and the immune system}

In normal conditions, consumed proteins, thus including food allergens, are completely degraded in the digestive tract to oligopeptide fragments. Due to intes-

Address for correspondence: Jacek Gocki MD, PhD, Department of Allergology, Clinical Immunology and Internal Diseases, Ludwik Rydygier Collegium Medicum, Nicolaus Copernicus University, 75 Ujejskiego St, 85-168 Bydgoszcz, Poland, phone: +48 603 171 732 , e-mail: jacekgocki@poczta.onet.pl

Received: 30.07.2015, accepted: 18.09.2015. 
tinal proteolytic enzyme activity, the latter are broken to di- and tripeptides and amino acids, and then absorbed by enterocytes. Further proteolysis takes place in enterocytes, to amino acids and dipeptides which enter portal circulation and then are carried to the liver. It turns out, however, that about $15 \%$ of consumed protein is incompletely digested [2], including a proportion of food allergens. Certain amounts of food antigens, which were not destroyed by digestion with enzymes, bile salts, and low gastric $\mathrm{pH}$, penetrate the epithelium of the digestive tract and reach the body's internal environment [3]. There are three pathways for food antigens to penetrate the digestive tract epithelium. The first involves the capture of antigens by Peyer's patch M cells. The second involves the capture of antigens from the digestive tract by the dendritic cell processes localized between enterocytes. The third involves the capture of antigens by enterocytes $[3,4]$. Food allergens may also permeate between enterocytes when they are damaged and the connections between them weakened, for instance due to inflammatory processes. Having penetrated the digestive tract epithelium, food antigens encounter cells of an immunocompetent system, the gut-associated lymphoid tissue (GALT). The role of GALT is to maintain the immune homeostasis between defending the organism from pathogens which have penetrated from the digestive tract, and inducing and maintaining the immune tolerance for innocuous antigens [3]. Therefore, food allergens will be treated by GALT either as innocuous antigens and induce tolerance, or as pathogens and then cause either defence reactions or excessive defensive reactions, that is hypersensitivity.

\section{The role of IgGs in inducing and maintaining the tolerance of food antigens}

The key role in inducing and maintaining of food allergen tolerance is played by induced Treg cells (iTreg). Specialized dendritic cells are also involved in this process. One type of them (CD103+) is responsible for migration to the lymph nodes, while the other (CX3CR1+) is non-migratory and subepithelial [4-6]. CD103+ cells in the lymph nodes induce the differentiation of naive T cells toward iTreg [7]. These cells (iTreg) migrate to the lamina propria of the intestinal epithelium, where they react with the CX3CR1+ dendritic cells, which results in the expansion of antigen-specific iTregs and suppression of allergic response involving the suppression of the Th2/ Th1 balance, release of IL10, suppression of effector cells (mastocytes, eosinophils, and basophils), and suppression of IgE synthesis, while inducing the production of IgG4 and IgA [4]. Thus it can be said that the increase in antigen-specific IgG 4 directed against food antigens is associated with the process of immune tolerance of these foods. Whereas food allergy is associated with the disturbance in or loss of immune tolerance to foods [4]. In patients with IgE-mediated food allergy, increased pro- duction of allergen-specific IgE antibodies is observed as well as low production or lack of allergen-specific lgG1 and IgG4 antibodies [8, 9]. The allergen-specific immunotherapy of food allergy seeks to change the immune response to a food antigen from allergy to its tolerance. Studies have shown that oral immunotherapy is very often associated with a reduction in slgE and an increase in slgG4. The increase in allergen-specific IgG4 during immunotherapy was related with inhibiting the bonding of slgE with their specific receptors and suppressing the activation of basophils [4].

\section{The role of IgG cells in triggering defence reactions to food allergens}

IgG antibodies comprise 70-75 immunoglobulins in the serum and are the fundamental antibodies of secondary immune response. Four subclasses of immunoglobulin $\mathrm{G}$ are distinguished: the $\operatorname{lgG} 1, \lg \mathrm{g} 2, \operatorname{lgG} 3$, and IgG4 subclasses amount to $66 \%, 23 \%, 7 \%$, and $4 \%$ of the IgG antigen pool, respectively. The IgG1-3 immunoglobulins are able to activate the complement, while the IgG4 do not show such abilities. The IgG antibodies are the main line of acquired defence and a body's specific humoral response to pathogens [10]. In normal conditions the digestive tract epithelium is impermeable to antigens, whereas when it is damaged by inflammatory processes antigens can permeate under the epithelium and contact immune system cells, which leads to immunization and production of specific defensive IgG antibodies. The subsequent contact of these antibodies with the antigen causes defence reactions involving the creation of antigen-antibody immune complexes, activation of complement protein cascade and effector cells, such as neutrophils, lymphocytes, macrophages, as well as eosinophils and platelets. As a result the immune complexes are phagocytosed and then destroyed in the reticuloendothelial system. Simultaneously, the inflammatory process caused by the immune reaction between slgG and food antigens might facilitate further damage and increased permeability of the digestive tract mucosa to food antigens. Therefore, the presence of specific IgG antibodies directed against food allergens reflects natural defence reactions of a body to allergens penetrating due to the damage of the epithelial barrier. Perhaps the IgG response to food allergens reflects the damage to the mucosa and develops secondary to it, and is also associated with the removal from the body of food antigens, which have accidentally penetrated the barrier of the mucosa, while the selectivity of response to certain food allergens may come from the type and quantity of a penetrating allergen and its resistance to digestion. This concept is well supported by the results obtained by Zuo et al. [11] who investigated the concentrations of slgG against 14 food allergens in patients with irritable bowel syndrome and functional dyspepsia, compared 
to a group of healthy patients. In all patients from both studied groups as well as in controls, the presence of slgG antibodies directed against food antigens was confirmed. Nevertheless, statistically significantly higher levels were observed in patients with irritable bowel syndrome and dyspepsia than in controls. Simultaneously, the authors stress the selectivity of response to only some food antigens, which may be associated with dietary habits or other factors. The same study did not reveal any correlation between the severity of the symptoms of functional dyspepsia and irritable bowel syndrome and slgG levels, while the total IgE concentrations in controls and studied groups did not differ statistically and were within normal ranges [11].

\section{The role of IgG antibodies in food allergy}

Food allergy is an immunologically conditioned, abnormal reaction to food allergens. While the reactions mediated by specific IgG antibodies directed against food antigens are immune reactions in nature, and therefore meet the first condition of food allergy, they are still normal reactions associated with the exposure to food antigens, and thus the second condition of the food allergy definition is not met.

In the light of current knowledge, the IgG-mediated reactions to food antigens indicate a repeated exposure to nutrients, which are recognized by the body's immune system as pathogens. The presence of slgG antibodies cannot be discussed in terms of a factor leading to hypersensitivity reactions but rather as a marker of immune tolerance associated with the activation of regulatory T cells. This is why laboratory tests based on the titrations of slgG against foods should be considered as insignificant in the diagnosis of food allergy and intolerance, and should not be performed in cases of symptoms associated with food consumption [12]. In a study carried out on 12 healthy volunteers, slgG4 against 9 commonly consumed foods were titrated (milk, eggs, peanuts, wheat flour, banana, orange, rice, potato, and pork). The slgG4 against the studied foods were found to be present in all participants, yet none of them reported any symptoms after having consumed the studied products, which suggests that these antibodies are produced as part of natural exposure to food [12].

In another study carried out on a group of children allergic to ovalbumin, a group of children with a resolved ovalbumin allergy, and a control group, IgG, IgG1, and IgG4 against ovalbumin were titrated. No statistically significant differences were found in the levels of investigated antibodies between the groups. In all groups the mean lgG value was $10 \mu \mathrm{g} / \mathrm{ml}$, with higher mean levels of lgG4-class antibodies $(10 \mu \mathrm{g} / \mathrm{ml})$ than $\mid \mathrm{gG} 1(1 \mu \mathrm{g} / \mathrm{ml})$. The researchers drew the conclusion that a strong IgG-mediated response is a normal physiological response to a frequently consumed protein [13].
The diagnosis of food allergy based on the titration of specific IgG antibodies against food allergens is often performed when it is impossible to explain the patient's complaints using the classical methods of diagnosis of IgE and non-lgE-mediated food allergy, while the patient is convinced that it was caused by the consumed foods.

Hochwaller et al. decided to investigate this problem by titrating slgG in subclasses $1-4$ and specific lgE against caseins and beta lactoglobulin, as well as total IgE, in the following patient groups: 1) with an IgE-mediated allergy to cow's milk protein, 2) with a confirmed intolerance of cow's milk proteins, 3) with gastrointestinal disturbances, and 4) in healthy persons. All patients with the exception of healthy persons reported various symptoms not limited to the gastrointestinal tract, while the patients from the first two groups clearly associated the complaints with the consumption of cow's milk. In the course of the study, the authors determined that there were no differences in slgG levels in subclasses 1-4 between the patients intolerant to cow's milk protein and the patients who tolerated it [14]. In addition, they noticed that only the patients with IgE-mediated allergy to milk had high levels of IgG1 and IgG4. IgG4 was higher in patients with a patent IgE-mediated allergy, whereas IgG2 and IgG3 were low in all studied groups. The patients intolerant to milk proteins had significantly lower levels of IgG compared to the patients with IgE-mediated allergy, and did not differ from persons tolerant to milk from the healthy group [14].

Antico et al. examined 73 patients reporting with skin symptoms who associated them with the consumption of foods. The reported symptoms were rash, itching of the skin, and erythema. All patients were subjected to skin tests for food and inhalation allergens, titrations of slgE and slgG4, open oral provocation challenges with foods for which slgG were detected, and double-blind placebocontrolled food challenges (DBPCFC) with foods for which the open food challenges were positive. In 38 patients, only slgG4 against foods were found, and both slgG4 and slgE were found in 7. In the remaining 28 patients no presence of slgG against foods was confirmed. In the DBPCFC trial, no patient out of the 45 in whom slgG 4 was found showed intolerance to the foods for which slgG4 was detected. In conclusion, the authors stated that titrating slgG4 in adult patients is not useful clinically in the diagnosis of food allergy or intolerance. The titration of slgG4 should not be a part of the diagnosis and therapy of adult patients with allergy-related skin disorders [15].

To summarize the relations between the slgG and slgE antibodies, it can be said that high slgG4 values are associated with asymptomatic sensitization and effective immunotherapy, which is indicative rather of a protective or blocking role of these antibodies [16]. Children with a high slgG4 to slgE ratio tolerate the sensitizing foods better [17]. High slgG in children with IgE-mediated allergy is a predictive factor of a future tolerance [18]. 


\section{The role of antibodies according to experts}

Expert committees of international scientific societies have also spoken on the role of IgG antibodies in the diagnosis and therapy. The European Academy of Allergology and Clinical Immunology (EACCI) issued a statement regarding food allergy, in which it stated that titrating IgG4 against foods is not recommended as a diagnostic tool. The presence of slgG 4 against foods indicates a repeated exposure to a food treated by the immune system as an alien protein and should not be treated as a sign of hypersensitivity but rather as a marker of immune tolerance associated with the activity of regulatory T cells. Specific IgG4 antibodies do not indicate food allergy or intolerance but a physiological response to the exposure to food [12]. The International Consensus ON (ICON) document on food allergy, prepared under the aegis of EAACl as well as the American Academy of Allergy, Asthma \& Immunology (AAAAI) and World Allergy Organization (WAO), clearly stresses that the titration of specific IgG against foods is not a recommended test in the diagnosis of food allergy [19]. In the practical guidelines for food allergy dated November 2014, prepared by a group of experts from the American Academy of Allergy, Asthma \& Immunology (AAAI), the American College of Allergy, Asthma \& Immunology (ACAAI), and the Joint Council of Allergy, Asthma \& Immunology (JCAAI), it is stated that tests based on the titration of allergen-specific IgG antibodies should not be used in the diagnosis of food allergy. Titrating allergen-specific IgG and IgG4 is not recommended in the diagnosis of non-lgE-mediated food allergies [20].

\section{Conflict of interest}

The authors declare no conflict of interest.

\section{References}

1. Mygind N, Dahl R, Pedersen S, Thestrup-Pedersen K. Alergologia. Kruszewski J, Silny W (Polish eds.) Urban \& Partner, Wrocław 1998; 136-7.

2. Majka J, Brzozowski T. Budowa i czynność jelita cienkiego. In: Wielka interna. Gastroenterologia. Dąbrowski A (eds). Medical Tribune Polska, Warsaw 2011; 2: 165-84.

3. Agarwal S, Mayer L. Mucosal immunity. In: Food Allergy: Adverse Reactions to Foods and Food Additives. 4th edition. Metcalfe DD, Sampson HA, Simon RA (eds). Blackwell Publishing, Oxford 2008; 19-29.

4. Tang MLK, Martino DJ. Oral immunotherapy and tolerance induction in childhood. Pediatr Allergy Immunol 2013; 24: 512-20.

5. Schulz O, Jaensson E, Persson EK, et al. Intestinal CD103+, but not CX3CR1+ antigen sampling cells migrate in lymph and serve classical dendritic cell functions. J Exp Med 2009; 206: 3101-14.

6. Bogunovic M, Ginhoux F, Helft J, et al. Origin of the lamina propria dendritic cell network. Immunity 2009; 31: 513-25.
7. Hadis U, Wahl B, Schulz O, et al. Intestinal tolerance requires gut homing and expansion of FoxP3+ regulatory cells in the lamina propria. Immunity 2011; 34: 237-46.

8. Beyer K, Castro R, Birnbaum A, et al. Human milk-specific mucosal lymphocytes of the gastrointestinal tract display a Th2 cytokine profile. J Allergy Clin Immunol 2002; 109: 707-13.

9. Flinterman AE, Pasmans SG, den Hartog Jager CF, et al. T cell responses to major peanut allergens in children with and without peanut allergy. Clin Exp Allergy 2010; 40: 590-7.

10. Male D, Brostoff J, Roth DB, Roit I. Immunologia. Żeromski J (Polish eds). Elsevier Urban \& Partner, Wrocław 2006; 59-86.

11. Zuo XL, Li YQ, Li WJ, et al. Alterations of food antigen-specific serum immunoglobulins $\mathrm{G}$ and $\mathrm{E}$ antibodies in patients with irritable bowel syndrome and functional dyspepsia. Clin Exp Allergy 2007; 37: 823-30.

12. Stapel SO, Asero R, Ballmer-Weber BK, et al. Testing for lgG4 against foods is not recommended as a diagnostic tool: EAACI Task Force Report. Allergy 2008; 63: 793-6.

13. Tay SS, Clark AT, Deighton J, et al. Patterns of immunoglobulin $\mathrm{G}$ responses to egg and peanut allergens are distinct: ovalbumin-specific immunoglobulin responses are ubiquitous, but peanut-specific immunoglobulin responses are up-regulated in peanut allergy. Clin Exp Allergy 2007; 37: 1512-8.

14. Hochwaller H, Schulmeister U, Swoboda I, et al. Patients suffering from non-lgE mediated cow's milk intolerance cannot be diagnosed based in IgG subclass or IgA responses to milk allergens. Allergy 2011; 66: 1201-7.

15. Antico A, Pagani M, Vescovi PP, et al. Food-specific lgG4 lack diagnostic value in adult patients with chronic urticaria and other suspected allergy skin symptoms. Int Arch Allergy Immunol 2011; 155: 52-6.

16. Wachholz PA, Durham SR. Mechanisms of immunotherapy: IgG revisited. Curr Opin Allergy Clin Immunol 2004; 4: 313-8.

17. Noh G, Ahn HS, Cho NY, et al. The clinical significance of food specific atopic dermatitis. Pediatr Allergy Immunol 2007; 18: 63-70.

18. Tomicic S, Norrman G, Falth-Magnusson K, et al. High levels of IgG4 antibodies to foods during infancy are associated with tolerance to corresponding foods later in life. Pediatr Allergy Immunol 2008; 20: 35-41.

19. Burks AW, Tang M, Sicherer S, et al. ICON: food allergy. J Allergy Clin Immunol 2012; 129: 906-20.

20. Sampson HA, Aceves S, Bock SA, et al. Food allergy: a practice parameter update - 2014. J Allergy Clin Immunol 2014; 134: 1016-25. 